\title{
Terahertz Time-Domain Spectroscopy Study of the Conductivity of Hole- Transporting Materials
}

\author{
Jan C. Brauer $\S^{\star}$, Verner K. Thorsmølle, and Jacques-E. Moser \\ §SCS Poster Prize Winner
}

\begin{abstract}
Some redox-active ionic liquids, organic amorphous solids containing electron-donating moieties, and conductive polymers can efficiently transport positive electrical charges. These hole-conducting media find increasing applications in unconventional solar cells and organic light-emitting diodes. Appropriate methods are required to unravel the detailed conduction and trapping mechanisms in these materials as well as to fully understand the interplay of molecular vibrations and charge transport processes. Here we present terahertz timedomain spectroscopy (THz-TDS) as a powerful technique, that allows the direct determination of the complex conductivity of hole transporting materials in a contactless, purely optical manner. Beyond the measurement of the conductivity of solid and liquid materials, $\mathrm{THz}$ (far infrared) spectroscopy also provides direct information of the librational and vibrational modes coupled to charge transport processes and therefore is invaluable in the study of the mechanism of polaronic transport in matter. Application of this technique is illustrated by examples provided by the study of the ionic liquid 3-methyl-1-propylimidazolium bis(trifluoromethane) sulfonimide (PMIT $\mathrm{FSI}$ ) and that of the molecular liquid hole conductor 10-methylphenoxazine. Both systems are of particular interest, as both types of holetransporting media are successfully used as alternatives to solvent-based electrolytes in dye sensitized solar cells
\end{abstract}

Keywords: Electrical conductivity · Electron transfer · Far IR spectroscopy · Hole-transporting materials · Terahertz time-domain spectroscopy

\section{Introduction}

The conducting and semiconducting properties of organic materials, such as ionic liquids, molecular liquids, glasses and polymers have attracted an increasing amount of attention during the past decades due to their possible applications in photoactive systems like light-emitting diodes and photovoltaic cells and in electronics. The main advantages of these materials over conventional semiconductors include low cost, easy processing and the possibility to produce flexible devices.
${ }^{*}$ Correspondence: J. C. Brauer Photochemical Dynamics Group,

Institute of Chemical Sciences \& Engineering Ecole Polytechnique Fédérale de Lausanne

EPFL SB ISIC GR-MO, Station 6

$\mathrm{CH}-1015$ Lausanne

E-mail: jancornelius.brauer@epfl.ch
A tremendous increase of the worldwide energy consumption and decreasing resources of fossil energy urges the research for alternative energy sources and for energy-saving devices. Dye-sensitized nanocrystalline solar cells (DSCs) ${ }^{[1,2]}$ and excitonic organic solar cells ${ }^{[3,4]}$ are believed to be cheap and sustainable alternatives to silicon and thin-film photovoltaics, whose energy pay-back time may exceed several years. Sealing of DSCs containing an organic solvent-based electrolyte is, however, a potential source of stability problems and represents an important fraction of the total cost of production of such devices. Hole-transporting materials (HTM) that allow the conduction of positive electrical charges usually by a hopping mechanism through an amorphous solid are already successfully employed in organic light-emitting diodes (OLEDs), whose commercial applications in displays and TV screens are growing rapidly. Likewise, non-volatile ionic liquids and organic charge-transporting solid materials like spiro-OMeTAD are viewed as interesting replacements to solvent-based electrolytes in DSCs. ${ }^{[2,5]}$. Imidazolium-based ionic liquids containing the redox couple $\mathrm{I}_{3}^{-/} \mathrm{I}^{-}$ have proved to be particularly interesting and power conversion efficiencies of up to $8.2 \%$ have been achieved. ${ }^{[6]}$
The optimization of organic conducting and semiconducting materials for the use in diodes and photovoltaic cells requires the origin and the nature of charge-transport processes in organic materials to be unravelled and the fundamentals of the optical, the electronic properties and the underlying photo-physics to be understood.

Conventionally such studies are performed using ac- and dc-conductivity measurements, ${ }^{[7]}$ time of flight experiments ${ }^{[8]}$ and optical pump-probe spectroscopy. In contrast to the first of those experimental methods, linear terahertz time-domain spectroscopy (THz-TDS) is a contactless, coherent, all optical technique, that allows direct determination of the complex conductivity of the probed material. Because of its ability to resolve mechanisms on the timescale of nuclear and electronic motion, time-resolved optical pump-probe spectroscopy is particularly interesting for scrutinizing spin ${ }^{[9]}$ and charge dynamics. ${ }^{[10,11]}$ Optical pump-THz probe spectroscopy ${ }^{[10]}$ allows one to observe induced changes in the conductivity as well as to determine if free charge carriers are formed directly or if they are formed via dissociation of excitons. Therefore, THz-TDS is an invaluable tool which brings additional insight into the fundamental nature of charge transport in such materials. In particular, the sensi- 
tivity of THz-TDS to free charges and low frequency vibrations offers the possibility to probe the possible coupling between those vibrations and the moving charges giving rise to polaronic transport.

The first applications of THz-TDS were in the domain of physics where it was used to study bound states such as excitons in semiconductors, ${ }^{[12]}$ Cooper pairs in superconductors ${ }^{[13]}$ and low energy transitions in metal oxides. This technique has also been applied in many other fields, such the analysis of gases by rotational spectroscopy, ${ }^{[14]}$ and the dynamics of liquids ${ }^{[15]}$ and biological molecules.[16] $\mathrm{THz}$ spectroscopy has also been successfully used to determine the efficiency of the formation of excitons and charge carriers in polymers. ${ }^{[17]}$

\section{Terahertz Time-Domain Spectroscopy}

THz-TDS is a nondestructive and noncontact technique which provides direct access to both the imaginary and the real part of the optical refractive index of the probed material. While the real part of the refractive index is directly related to the speed of light in matter, the imaginary part gives straight-forward information on light absorption in the far infrared and therefore information about active lowfrequency vibrational modes. The complex refractive index can also be directly translated in terms of complex electrical conductivity of carriers in the material. THz-TDS bridges the gap between midinfrared spectroscopy and microwave techniques; it covers the range from 100 $\mathrm{GHz}$ to several $\mathrm{THz}$. Converted to other units $1 \mathrm{THz}$ corresponds to $33.33 \mathrm{~cm}^{-1}$, $4.16 \mathrm{meV}$, or $300 \mu \mathrm{m}$. This spectral region in the far-infrared wavelength domain has been difficult to access due to technical problems which have been overcome by using femto-second laser pulses for the generation and detection of $\mathrm{THz}$ radiation. ${ }^{[18]}$

There are several methods to generate $\mathrm{THz}$ radiation by using photo-conducting antennas, planar antennas or optical rectification. Because optical rectification is the difference frequency mixing analogue to second harmonic generation, the bandwidth of the optical pulse determines the accessible $\mathrm{THz}$ frequency range. For ultra short laser pulses, with a broad frequency bandwidth, all the frequency components are differenced with each other, producing a pulse with frequencies from 0 to several THz. To obtain a broad $\mathrm{THz}$ spectrum a broad spectral width of the optical pulse is needed, which is accomplished by using femtosecond pulses. The advantage of optical rectification is that the $\mathrm{THz}$ pulse width depends only on the duration of the

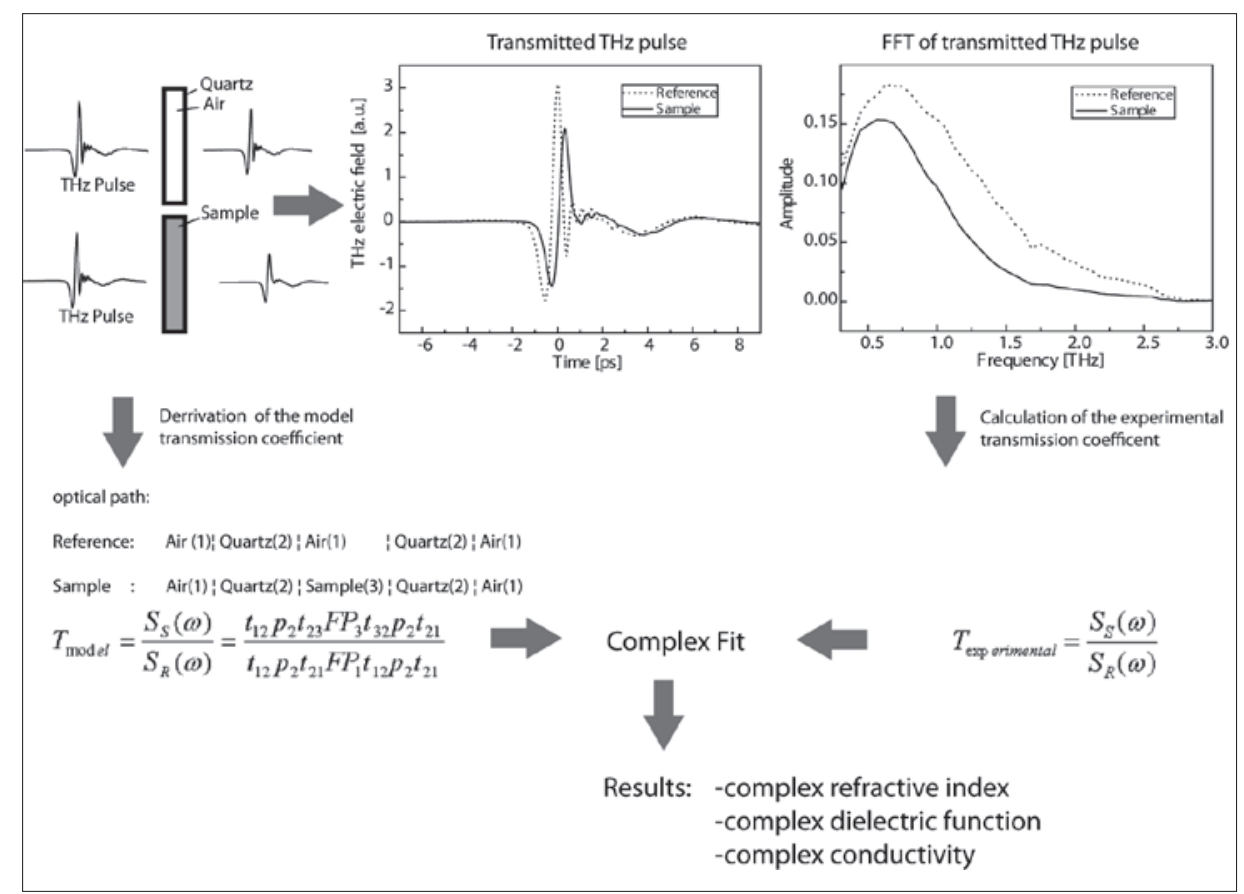

Fig. 1. Schematic representation of the data acquisition and analysis in linear THz-TDS.

optical pulse and on the phonon absorption modes of the crystal.

The detection of THz pulses is usually done by free space electro optic sampling (FSEOS). This technique is based on the Pockels effect, in which the electric field of the $\mathrm{THz}$ pulse induces birefringence in a detector crystal. If an optical gating pulse travels through the detector crystal at the same time as the THz pulse, its polarization is rotated. This rotation of the polarization is proportional to the magnitude of the THz electric field. By varying the delay of a visible light gating pulse the $\mathrm{THz}$ electric field can be mapped out in the time domain.

In our setup ${ }^{[19]}$ a chirped pulse amplified Ti:Sapphire laser system provides $120 \mathrm{fs}$ pulses at a $1 \mathrm{kHz}$ repetition rate and at a central wavelength of $778 \mathrm{~nm}$. One part of the beam is used to generate nearly single-cycle $\mathrm{THz}$ pulses via optical rectification in a $\mathrm{ZnTe}$ crystal. The $\mathrm{THz}$ pulse is focused on the sample and the transmitted pulse is refocused on the $\mathrm{ZnTe}$ detector crystal by gold mirrors. The other part of the initial laser beam is used as a gating beam and guided over a delay line onto the detector crystal. The birefringence induced by the THz electric field rotates the polarization of the gating beam in a way that it is elliptically polarized after a quarter wave plate. The two polarization components are then separated and subtracted by a pair of balanced photo detectors. The signal of the photodiodes is then measured by a lock-in amplifier referenced to a chopper wheel placed in front of the generator ZnTe crystal.

\section{Extraction of the Complex Parameters by THz-TDS}

Recording a $\mathrm{THz}$ time-domain spectrum consists of two measurements, one of the sample and one of a reference with known parameters, which have to be carried out under exactly the same conditions. A typical THz-TDS signal is shown in Fig. 1.

One of the unique characteristics of THz-TDS is that not only the amplitude but also the phase shift is measured after having passed through the sample material. This allows the simultaneous determination of the refractive index and the absorption coefficient without any approximations, assumptions or the use of the KramersKronig analysis. In Fig. 1 one notices that the sample signal is retarded with respect to the reference signal and its amplitude is decreased. The time delay between the reference and the sample signal gives an estimate of the refractive index:

$$
\Delta t=\frac{\left(n-n_{r e f}\right) L}{c}
$$

where $n$ is the refractive index, $L$ the optical path in the sample material, $\Delta t$ the time delay and $c$ the velocity of light in vacuum.

The procedure to extract the complex conductivity, the complex dielectric function, the refractive index and the absorbance in outlined in the following. The general idea is to fit the experimentally determined transmission coefficient to a 
model transmission coefficient taking into account the transmission and reflection at each interface, as well as the propagation and multiple reflections inside the media.

After appropriate time windowing and zero padding, the Fast Fourier Transform (FFT) of the sample signal is divided by the FFT of the reference signal to obtain a frequency-dependent transmission coefficient. The model transmission coefficient is derived by using the Fresnel equations for reflection and transmission. Fig. 1 illustrates the path of the light and particularly the different interfaces that have to be taken into account to develop an expression for the model transmission coefficient. An expression for the model transmission coefficient for a quartz cuvette is given in Fig. 1. $t$. represents the Fresnel transmission coefficients at the interface between medium $i$ and $j$, and $p_{\mathrm{j}}$ and $F P$ are the propagation coefficient and a Fabry-Perot interference term in medium $j$, respectively. The last term has to be added to account for multiple reflections when the optical path in the cuvette is too small to allow for a temporal separation between the directly transmitted pulse and its reflections inside the cuvette.

Calculation of all terms of this expression requires a value of the complex refractive index $\tilde{n}$ of the sample. This is evaluated by performing a complex fit of the model transmission coefficient to the experimental one.

Having extracted the complex refractive index $\tilde{n}=n+i \kappa$, the dielectric function $\varepsilon$, the conductivity $\sigma$ and the absorption constant $\alpha$ can be calculated from the following expressions. Importantly, it is noted that the real part of the conductivity differs from the absorption constant only by a constant factor.

$$
\tilde{\varepsilon}=\varepsilon^{\prime}+\varepsilon^{\prime \prime}=n^{2}-\kappa^{2}+i 2 n \kappa
$$

$$
\begin{aligned}
\tilde{\sigma}=\sigma^{\prime}+\sigma^{\prime \prime}= & 2 n \kappa \in_{0} \omega+i \in_{0} \\
& \omega\left(1-n^{2}+\kappa^{2}\right)
\end{aligned}
$$

$$
\alpha=\frac{2 \omega \kappa}{c}
$$

where $\tilde{\varepsilon}$ is the complex dielectric function with $\varepsilon$ ' and $\varepsilon$ " being the real and imaginary part respectively, $n$ is the refractive index, $\kappa$ the extinction coefficient. $\tilde{\sigma}$ is the complex conductivity with $\sigma$ ' and $\sigma$ " being the real and imaginary part, respectively, $\epsilon_{0}$ the permittivity of free space, $\omega$ the angular frequency, and $c$ the speed of light.

\section{Examples}

The application of the THz-TDS technique to the case of two different liquid holeconducting media is illustrated here. Fig. 2A displays the real and imaginary part of the conductivity of a melt of pure 3-methyl-1propylimidazolium bis(trifluoromethane) sulfonimide (PMITFSI) ionic liquid, as extracted from the raw $\mathrm{THz}$ temporal signal. The real part of the conductivity is seen to increase monotonically with increasing frequency; behavior which is characteristic of a disordered or localized system with no long range order such as in a liquid. As the frequency increases, the length scale for carrier displacement becomes shorter and for a disordered system the conductivity increases. Therefore the conductivity can certainly not be described by a free electron model, like the Drude Model[20] developed to describe the electronic conduction in metals and predicting a decrease of conductivity for increasing frequencies. A negative imaginary conductivity as observed for PMITFSI at frequencies below 1.7 THz could be explained by the DrudeSmith model. ${ }^{[21]}$ Additionally, the conductivity spectrum of PMITFSI shows a broad peak at about $2 \mathrm{THz}$ that is indicative of an absorption resonance. The associated vibrational mode (at $\tilde{v}=66.7 \mathrm{~cm}^{-1}$ ) was assigned to an oscillation of the distance separating contiguous anions and cations in the ionic liquid. A detailed study of this particular feature, as well as that of electrical conduction mechanisms in redoxactive ionic liquids containing iodide and triiodide at various concentrations and temperatures will be reported elsewhere.

Fig. 2B shows the real conductivity obtained from THz-TDS of 10-methylphenoxazine, a liquid hole transporter. Spectra are provided for the pure molecular compound, as well as for the liquid containing two different concentrations of an oxidizer. As in PMITFSI the conductivity spectrum shows an increase of conductivity with increasing frequency that is characteristic for disordered systems. The conductivity spectrum of the pure 10-methylphenoxazine is almost flat. Comparing the conductivities of pure PMITFSI and pure 10-methylphenoxazine at $0.3 \mathrm{THz}$ one notices that the conductivity of the ionic liquid is about one order of magnitude higher than that of the liquid hole conductor. While in PMITFSI the conductivity is ensured by diffusion of ions, the absence of charge carriers in the neutral molecular liquid makes this medium an insulator. By oxidizing the 10-methylphenoxazine with $\mathrm{NOBF}_{4}$, stable radical cations of the compound are formed, which are thought to contribute to the conductiv-

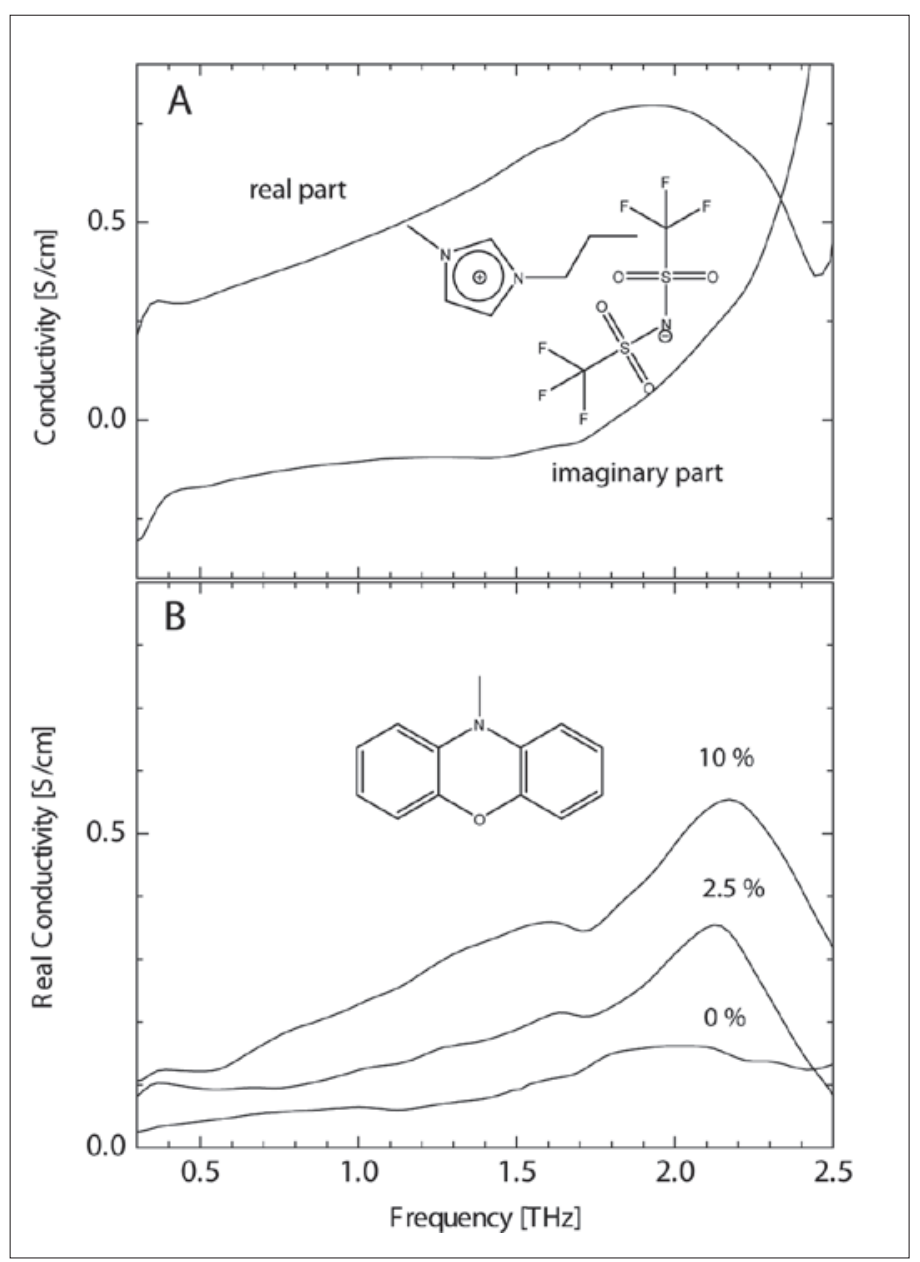

Fig. 2. Real and imaginary parts of the conductivity extracted from $\mathrm{THz}-$ TDS measurements applied to $(A)$ PMITFSI, and (B)10methylphenoxazine. In the latter case, results for a sample of the pure liquid and for samples doped with $2.5 \%$ and $10 \%$ of $\mathrm{NOBF}_{4}$ are shown. 
ity by a hopping mechanism. The conductivity increases proportionally to the concentration of holes and thus increases with chemical oxidation (p-doping). In addition, two resonance features are observed at 1.6 $\mathrm{THz}$ and $2.15 \mathrm{THz}$ in the partially oxidized sample and tend to be more pronounced for larger degrees of doping. The very small resonance peaks found in the conductivity spectrum of the undoped sample likely result from residual oxidation due to contact with air. The associated vibrational modes are therefore assigned to the phenoxazine radical cation. The geometries of the neutral phenoxazine molecule and its radical cation are quite different. The neutral molecule adopts a highly non-planar configuration bent on the line connecting the nitrogen and the oxygen, while the radical cation has a more planar structure.[22] A nuclear reorganization by bending and stretching of the bonds as well as a reorganization of the surrounding molecules has to take place prior to the actual charge transfer in the hopping event in order to achieve electronic resonance between reactant and product state. The activation energy of this process can be obtained, according to the Marcus theory, from the intersection of the free energy curves as a function of nuclear coordinates of the donor and acceptor molecule. One could imagine testing whether the observed vibrational modes in the conductivity spectrum contribute to the population of the transition state by performing THz-pump $\mathrm{THz}$ probe experiments. Besides the internal reorganization energy, the electronic coupling between two neighboring molecules plays an important role for the rate of charge transfer in such systems.

\section{Acknowledgements}

The PMITFSI ionic liquid was kindly provided by the Laboratory of Photonics and Interfaces (LPI) of EPFL. Fruitful discussions in particular with Dres S. M. Zakeeruddin and G. Rothenberger, as well as experimental help by J. Teuscher are gratefully acknowledged. This research is made possible thanks to the financial support of the Swiss National Science Foundation and scientific equipment funding by EPFL.

Received: January 14, 2009

Revised: March 12, 2009

[1] B. O'Regan, M. Grätzel, Nature 1991, 353, 737.

[2] D. Kuang, C. Klein, Z. Zhang, S. Ito, J.-E. Moser, S. M. Zakeeruddin, M. Grätzel, Small 2007, 3, 2094.

[3] B. Fan, R. Hany, J.-E. Moser, F. Nüesch, Org. Electronics 2008, 9, 85 .

[4] B. A. Gregg, J. Phys. Chem. B 2003, 107, 4688.

[5] U. Bach, D. Lupo, P. Comte, J.-E. Moser, F. Weisörtel, J. Salbeck, H. Spreitzer, M. Grätzel, Nature 1998, 395, 583.

[6] Y. Bai, Y. Cao, J. Zhang, M. Wang, R. Li, P. Wang, S. M. Zakeeruddin, M. Grätzel, Nature Mater. 2008, 7, 626.

[7] L. Leontie, I. Druta, B. Furdui, G. I. Rusu, Prog. Org. Coat. 2007, 58, 303.

[8] R. J. Bushby, O. R. Lozman, Curr. Opin. Solid. State Mater. 2002, 6, 569

[9] J. M. Kikkawa, I. P. Smorchkowa, N. Samarth, D. D Awschalom, Physica E 1998, 2, 394.

[10] R. D. Averitt, A. I. Lobad, C. Kwon, S. A. Trugman, V. K. Thorsmølle, A. J. Taylor, Phys. Rev. Lett. 2001, 87, 017401.

[11] J. S. Dodge, A. B. Schuhmacher, J.-Y. Bigot, D. S. Chemla, N. Ingle, M. R. Beasley, Phys. Rev. Lett. 1999, 83, 4650.

[12] M. C. Beard, G. M. Turner, C.A. Schmuttenmaer, J. Phys. Chem. B 2002, 106, 7146.

[13] J. Corson, R. Mallozi, J. Orenstein, J. N. Eckstein, I. Bozovic, Nature 1999, 398, 221.

[14] R. H. Jacobsen, D. M. Mittlemann, M. C. Nuss, Opt. Lett. 1996, 21, 2001.

[15] C. Rønne, L. Thrane, P.-O. Åstrand, A. Wallqvist, K. V. Mikkelsen, S. R. Keiding, J. Chem. Phys. 1997, 107, 5319.

[16] M. Walther, B. Fischer, M. Schall, H. Helm, P. U. Jepsen, Chem. Phys. Lett. 2000, 332, 389.

[17] E. Hendry, J. M. Schins, L. P. Candeias, L. D. A. Siebbeles, M. Bonn, Phys. Rev. Lett. 2004, 92 , 19.

[18] C. A. Schmuttenmaer, Chem. Rev. 2004, 104, 1759

[19] V. K. Thorsmølle, B. Wenger, J. Teuscher, J. C. Bauer, J.-E. Moser, Chimia 2007, 61, 631.

[20] P. K. L. Drude, Phys. Z. 1900, 1, 161.

[21] N. V. Smith, Phys. Rev. B 2001, 64, 155106.

[22] L. Yang, J.-K. Feng, A.-M. Ren, C.-C Sun, Polymer 2006, 47, 3229. 